\title{
Medieval Disputationes de obligationibus as Formal Dialogue Systems
}

\author{
Sara L. Uckelman
}

Published online: 17 March 2012

(C) The Author(s) 2012. This article is published with open access at Springerlink.com

\begin{abstract}
Formal dialogue systems model rule-based interaction between agents and as such have multiple applications in multi-agent systems and AI more generally. Their conceptual roots are in formal theories of natural argumentation, of which Hamblin's formal systems of argumentation in Hamblin (Fallacies. Methuen, London, 1970, Theoria 37:130-135, 1971) are some of the earliest examples. Hamblin cites the medieval theory of obligationes as inspiration for his development of formal argumentation. In an obligatio, two agents, the Opponent and the Respondent, engage in an alternating-move dialogue, where the Respondent's actions are governed by certain rules, and the goal of the dialogue is establishing the consistency of a proposition. We implement obligationes in the formal dialogue system framework of Prakken (Knowl Eng Rev 21(2):163-188, 2006) using Dynamic Epistemic Logic (van Ditmarsch et al. in Dynamic epistemic logic, Synthese Library Series. Springer, Berlin, 2007). The result is a new type of inter-agent dialogue, for consistency-checking, and analyzing obligationes in this way also sheds light on interpretational and historical questions concerning their use and purpose in medieval academia.
\end{abstract}

Keywords Consistency $\cdot$ Dialogue protocol $\cdot$ Dialogue systems $\cdot$ Obligationes

\section{Introduction}

Rule-based interactions such as dialogues or arguments are ubiquitous and diverse; they are the basic method of communication between agents. Such interactions are modeled in artificial intelligence and computer science by formal dialogue systems (FDSs) and dialogue games (Karunatillake et al. 2009; Maudet 2003; McBurney

S. L. Uckelman $(\bowtie)$

Tilburg Center for Logic and Philosophy of Science, Tilburg University, Dante Building,

PO Box 90153, 5000 LE Tilburg, The Netherlands

e-mail: S.L.Uckelman@uvt.nl 
and Parsons 2002; McBurney and Parsons 2009; Prakken 2006). These systems give formal, and hence potentially implementable, methods for modeling real-life dialogue situations, such as complex reasoning in legal domains. More generally, dialogue systems and games are used in multi-agent systems to model distributed cognition and interaction between intelligent agents, and they can also be used in the specification of complex software systems and programs (McBurney and Parsons 2009).

The conceptual roots of formal dialogues, however, come not from within AI but from without. They are located in the sphere of natural argumentation, that is, philosophical logic, argumentation theory, and rhetoric, and are "meant to provide formal structures to represent how a sequence of rational argumentation should proceed when one party argues with another in an orderly way" (Walton 2000, p. 329). One of the earliest attempts to provide a theory of formal dialogues is Hamblin (1970, 1971). In Hamblin (1970), Hamblin locates part of the motivation for his development of formal argumentation in historical formal dialogue systems, that is, dialogical or disputational settings where explicit rules are given governing the actions of the participants. One such system of natural argumentation that he considers in particular is the medieval theory of obligationes, developed in the thirteenth and fourteenth centuries. In an obligatio, two agents, the Opponent and the Respondent, engage in an alternating-move dialogue, where the Respondent's actions are governed by certain rules, and the goal of the dialogue is, in the most basic case, to establish the consistency of a proposition. We argue that obligationes are best modeled by FDSs because of their intrinsic dialogical nature. Furthermore, taking obligationes from the realm of natural argumentation to the realm of formal dialogue systems results in the determination of a new type of dialogue system different from those generally discussed in literature on argumentation, and thus they make a novel contribution to the formal modeling of dialogue and interaction.

The plan of the paper is as follows. In Sect. 2 we present the medieval theory of obligationes, focusing specifically on the works of one author, Walter Burley, and give examples. In Sect. 3 we briefly survey previous work on obligationes, both formal and philosophical, and motivate modeling obligationes as FDSs by showing how they can make sense of King's interpretation of obligationes as a metadisputational framework. In Sect. 4 we introduce formal dialogue systems, following the presentation in Prakken (2006), and show generically how obligationes can be viewed as a formal dialogue system. Before we give a precise specification of obligationes as FDSs in Sect. 6, we first outline the logic used in the argumentation, a type of Dynamic Epistemic Logic, in Sect. 5. We discuss the properties of the protocol we introduce, compare our results with standard types of FDSs, and define a new type of inter-agent dialogue, for consistency- or feasibilitychecking, in Sect. 7. In Sect. 8 we conclude and point towards future work.

\section{Medieval Theories of obligationes}

An obligatio is a dialogue between two agents, the Opponent and the Respondent, where the Opponent puts forward a sequence of propositions, and the Respondent is 
obligated (hence the name) to follow certain rules in his responses to the Opponent's propositions. More precisely, the Opponent puts forward an initial statement, called the positum, which the Respondent can either admit or refuse to admit it. If he admits it, the obligatio begins. If he does not, no obligatio begins. If the obligatio begins, the Opponent puts forward propositions and the Respondent has three ways that he can respond: He can grant or concede the proposition, he can deny the proposition, or he can doubt it, where 'doubt' should be understood as 'remain agnostic about'; doubting $\varphi$ does not entail any commitment to $\neg \varphi$. (Some authors, such as Ockham (1974) and the anonymous author of the Obligationes Parisienses (de Rijk 1975), mention a fourth option, which is to 'draw distinctions', that is, to clarify an ambiguity on the part of the Opponent.) The obligatio continues until the Opponent calls "Cedat tempus" ("Time's up"), whereupon the responses of the Respondent are analysed with respect to the Respondent's obligations, to determine whether he has responded well or badly.

The earliest texts on obligationes date from the beginning of the thirteenth century (de Rijk 1974, 1975, 1976), and many of the leading logicians from that century and the next wrote treatises on the subject. While the roots of obligational disputations are clearly grounded in Aristotle's discussion of dialectical exchanges in the Topics VIII, 4 (159a15-24) and in the Prior Analytics I, 13 (32a18-20) (cf. (Yrjönsuuri 1994 §II.A)), the systematic development of the theory of obligationes over the course of the 13th and 14th centuries tends to show little adherence to the Aristotelian tradition and definitions. While the specific details vary from author to author, a number of distinct types of obligationes discussed by multiple authors can be identified. The six most common are positio, depositio, dubitatio, sit verum or rei veritatis, institutio, and petitio. Of these six, positio is universally the most widely studied, both by medieval and modern authors; as a result, it is the focus of the current paper. For further information on obligationes, including a discussion of their purpose and their role in medieval philosophy, see Uckelman (2012); Yrjönsuuri (1994).

To make the above more precise, we look at the theory of obligationes of a specific writer, Walter Burley. Burley's treatise De obligationibus, written around 1302, gives a standard treatment of positio. The text of this treatise is edited in Burley (1963) and a partial translation of the text, including the section on positio in its entirety, is found in Burley (1988). Burley defines the general goal of an obligatio as follows:

The opponent's job is to use language in a way that makes the respondent grant impossible things that he need not grant because of the positum. The respondent's job, on the other hand, is to maintain the positum in such a way that any impossibility seems to follow not because of him but rather because of the positum (Burley 1988, p. 370). ${ }^{1}$

\footnotetext{
1 "Opus opponentis est sic inducere orationem ut faciat respondentem concedere impossibilia quae propter positum non sunt necessaria concedere. Opus autem respondentis est sic sustinere positum ut propter ipsum non videatur aliquod impossibile sequi, sed magis propter positum. Igitur intentio opponentis et respondentis circa enuntiabile versatur ad quod respondens est obligatus" (Burley 1963, p. 34).
} 
Thus, it is clear that in an obligatio, the goal is consistency, not logical truth or validity. In positio, the primary obligation of the Respondent is to grant, that is, to hold as true, the positum. If the Respondent accepts the positum and the obligatio begins, he is obliged to follow the following rules:

1. Everything that is posited and put forward in the form of the positum during the time of the positio must be granted (Burley 1988, p. 379). ${ }^{2}$

2. Everything that follows from the positum must be granted. Everything that follows from the positum either together with an already granted proposition (or propositions), or together with the opposite of a proposition (or the opposites of propositions) already correctly denied and known to be such, must be granted (Burley 1988, p. 381). ${ }^{3}$

3. Everything incompatible with the positum must be denied. Likewise, everything incompatible with the positum together with an already granted proposition (or propositions), or together with the opposite of a proposition (or the opposites of propositions) already correctly denied and known to be such, must be denied (Burley 1988, p. 381). ${ }^{4}$

In Rule 1, 'in the same form as' should be understood syntactically; if the positum is 'Marcus is Roman', then the Respondent doesn't have an obligation to accept 'Tullius is Roman' unless it is explicit (either through common knowledge or through previous concessions) that Marcus is Tullius. ${ }^{5}$ In Rules 2 and 3, the clause "known to be such" indicates a public (and hence testable) phenomenon; it is not a reference to the Respondent's knowledge alone, but to the common knowledge of the Respondent and Opponent.

Burley also defines a notion of relevance of propositions which applies to all types of obligatio. A proposition is irrelevant or impertinent if neither it nor its negation follows from the set of propositions which have already been conceded (which includes the negations of propositions which have been denied).

- Rule for Irrelevant Propositions One must reply to what is irrelevant in accordance with its own quality (Burley 1988, p. 375). ${ }^{6}$

\footnotetext{
2 "Omne positum, sub forma positi propositum, in tempore positionis, est concedendum" (Burley 1963, p. 46).

3 "Omne sequens ex posito est concedendum. Omne sequens ex posito cum concesso vel cum concessis, vel cum opposito bene negati vel oppositis bene negatorum, scitum esse tale, est concedendum" (Burley 1963, p. 48).

4 "Omne sequens ex posito est concedendum. Omne sequens ex posito cum concesso vel cum concessis, vel cum opposito bene negati vel oppositis bene negatorum, scitum esse tale, est concedendum" (Burley 1963, p. 48).

5 Burley says, "The clause 'put forward in the form of the positum' is used because if [something] is put forward in a form other than the form of the positum, it need not be granted. For example, if 'Marcus' and 'Tully' are names of the same man, and it is posited that Marcus is running, one need not grant that Tully is running" (Burley 1988, p. 379) ("Et ponitur haec particula: sub forma positi propositum, quia, si proponatur sub alia forma quam sub forma positi, non oportet quod concedatur. Ut si Marcus et Tullius sint nomina eiusdem, et ponatur Marcum currere, non oportet concedere Tullium currere" (Burley 1963, §C.1.a).).

6 "Similiter, ad impertinens respondendum est secundum sui qualitatem" (Burley 1963, p. 42).
} 
Table 1 An example obligatio

\begin{tabular}{llll}
\hline & Opponent & Respondent & \\
\hline 1 & $\varphi$ & I admit it & $\Phi_{0}:=\{\varphi\}$ \\
2 & $\neg \varphi \vee \psi$ & I grant it & $\Phi_{1}:=\{\varphi, \neg \varphi \vee \psi\}$ \\
3 & $\psi$ & I grant it & \\
\hline
\end{tabular}

I.e., the Respondent should reply by conceding the proposition if it is true, by denying if it is false, and by doubting if he does not know which is the case. The notion of 'relevance' in obligationes parallels the concept of 'support' in formal argumentation theory where an argument is defined as a pair $A=(S, p)$ where $S$ is a consistent subset of the knowledge base such that $S \models p$ (cf., e.g., (Cogan et al. 2006, p. 155$))^{7}$

Because 'I don't know' (or 'I doubt it', or 'Prove!' (Proba!)) is an acceptable answer in an obligational disputation, it is thus clear that the moves in an obligational disputation cannot be understood as the Opponent asking simple yes/no questions, since "[a] yes-no question admits of only two direct answers-the affirmative answer (yes) and the negative answer (no). A yes-no question is designed to rule out the option 'I don't know' as an answer or acceptable reply" (Walton 2000, p. 335).

A simple example illustrating Burley's rules for positio is given in Table 1. Suppose $\varphi$ does not imply $\neg \psi$ and $\varphi$ is known to be contingently false. In the first round, the Opponent puts forward a contingent (but false) proposition, $\varphi$; the Respondent grants it in accord with Rule 1. In the second round, either $\varphi$ implies $\psi$, then the sentence $\neg \varphi \vee \psi$ is relevant and follows from $\Phi_{0}$ (the set of propositions conceded so far along with the negations of propositions denied to this point); or it doesn't follow, in which case it is irrelevant and true (since $\varphi$ is false). In both cases, the Respondent is required to concede; the first case falls under Rule 2, and the second under the Rule for Irrelevant Propositions. In the third round, the Respondent likewise must concede because $\psi$ follows from $\Phi_{1}$. This example obligatio shows how, given a positum which is false but not contradictory, the Opponent can force the Respondent to concede any other consistent proposition.

More interesting examples, such as the example in Table 2, involve statements about the obligational rules themselves. Let $\varphi$ be the proposition 'you are in Rome' (spoken by the Opponent to the Respondent). The positum is a disjunction between a simple proposition and the assertion that that proposition must be granted. Because the disjunction is not a logical contradiction (in particular the first disjunct is possible, though it is in fact false), the Respondent is correct in accepting the positum. The second disjunct is irrelevant, as it is not a logical consequence of the positum, and furthermore it is false: Since $\varphi$ is false, and $\varphi$ is also irrelevant, the Respondent is not under any obligation to accept $\varphi$. Thus it is false that $\varphi$ must be

\footnotetext{
7 This definition of relevance was standard until Roger Swyneshed redefined the term in his Obligationes, written between 1330 and 1335; thereafter some authors followed Burley and others Swyneshed. See (Uckelman 2012, §4) for a full discussion of this.
} 
Table 2 A more interesting example

\begin{tabular}{lll}
\hline & Opponent & Respondent \\
\hline 1 & $\varphi$ or $\varphi$ must be granted & I admit it \\
2 & $\varphi$ must be granted & I deny it \\
3 & $\varphi$ follows from the positum and the opposite of something correctly denied & I grant it \\
4 & $\varphi$ must be granted & $? ?$ \\
\hline
\end{tabular}

granted, so he correctly denied the second proposition. The third proposition expresses a logical necessity, about the validity of disjunctive syllogism, and so is accepted. But now it is unclear how the Respondent should respond to the reassertion that $\varphi$ must be granted. On the one hand, this proposition has been put forward before, and was denied, and so it should continue to be denied. On the other hand, once the third proposition has been granted, by Rule 2, $\varphi$ must be granted. So superficially it appears that the Respondent is obliged to both accept and deny this final statement. Burley's resolution to the problem is to argue that (3) is not only not necessary, but it is repugnant, since it is inconsistent with the opposite of (2). Since it is repugnant, the Respondent should have in fact denied it, and thus (4) can also be denied without contradiction (Yrjönsuuri 1994, pp. 152-155).

\section{Previous Work on obligationes}

Green's Ph.D. dissertation, containing an edition of and commentary on two treatises on obligationes, now generally ascribed to William of Sherwood and Walter Burley, marks the beginning of modern research on obligationes. ${ }^{8}$ Since then, many philosophers and historians have devoted themselves to the question of the goal or purpose of obligational disputations and the role they played in medieval academic life, while somewhat fewer have focused on the logical properties of obligationes. Despite this, the purpose of obligationes and their role in medieval academic life remains stubbornly unclear (Spade 2000, 2008). Two recent sources which discuss the various views, which range from the view that obligationes were mere academic exercises, that they were used for counter-factual reasoning, that they were a tool for evaluating sophismata and insolubilia (paradoxical and problematic sentences), that they are thought experiments, and that they provide a theory of belief revision, are Yrjönsuuri (1994, 2001).

Hamblin is the first modern author to attempt to formalize obligationes (Hamblin 1970 , pp. 260-263). Given his interest in formalizing argumentation generally, he focuses on the dialogical aspects of obligationes. His formalization is rudimentary and models only one variant, that given by William of Sherwood, ${ }^{9}$ but it marks the

\footnotetext{
${ }^{8}$ While Boehner mentioned obligationes in the beginning of Boehner (1952), it wasn't until Green's dissertation that obligationes were researched widely or in any depth.

${ }^{9}$ Hamblin routinely questions the attribution to Sherwood of the text he is considering; however, more recent scholarship is agreed that the text was almost certainly written by Sherwood, sometime in the middle of the thirteenth century (Braakhuis 1998).
} 
beginning of modern scholarship on the formal properties of obligationes. Recent scholarship has focused on the game-like nature of obligationes, e.g., de Rijk (1975); Dutilh Novaes (2007); Yrjönsuuri (1994). In particular, there is an immediate apparent similarity between obligationes and Lorenzen's dialogical logic (Lorenzen and Lorenz 1978). It may therefore seem natural to look to game-based structures in logic to provide a general framework for modeling different types of obligationes. However, there are a number of aspects which do not immediately lend themselves nicely to a game-like interpretation (e.g., the notion of a winning strategy for an obligatio, for example, is difficult to define ${ }^{10}$; see Uckelman (2011b) for a further discussion of the issues comparing obligationes with Lorenzen dialogue games.), and despite the strongly logical component of obligationes, to date relatively little work has been done on the formal properties of the logic involved and few attempts have been made to provide an explicit specification of the game(s) involved.

The most extensive attempt is in Dutilh Novaes (2007). In this book, Dutilh Novaes analyses the obligational theories of three authors, Walter Burley, Richard Swyneshed (c.1330), and Ralph Strode (second half of the 14th C), giving separate formalizations for each one. Her formalizations are based on models $\mathfrak{M}=$ $\left\langle K_{c}, \Phi, \Gamma, R(\varphi)\right\rangle$ where $K_{c}$ is the set of common knowledge among the participants of the disputation (expressed as a set of propositions); $\Phi$ is a sequence of propositions, which keeps track of the assertions of the Opponent; $\Gamma$ is a sequence of propositions, which keeps track of the responses of the Respondent; and $R(\varphi)$ is a function from $\varphi$ to 1 (standing for 'concede'), 0 ('deny'), and ? ('doubt'). These formalizations are not very satisfying for a number of reasons. Each obligational theory studied is provided with a different framework, which means that it is difficult to make cross-theory comparisons. Further, only positio is studied; the other types of obligationes are not discussed. While in the present paper we also focus solely on positio, we have shown elsewhere (Uckelman 2011a) how the variant dubitatio can be treated within the framework defined in $\$ 5$. Additionally, Dutilh Novaes's frameworks all presuppose a significant amount of background information which is taken for granted and never specified: the semantic model(s) in which truth of propositions (particularly the positum and irrelevant propositions) and the Respondent's knowledge of both individual propositions as well as how the consequence relations are to be evaluated, and the syntactic rules governing $\vdash$ (which is used to generate $\Gamma$ ). For example, the set of common knowledge $K_{C}$ is not defined in any explicit fashion, and there is nothing which grounds the knowledge of the participants. Finally, since the nature of the proof system being used in the definition of $R(\varphi)$ is never specified, her frameworks are essentially incomplete; it is impossible to implement the logical model without making the proof-system explicit (Dutilh Novaes 2007, p. 169).

When looking for an alternative to a game-theoretic approach to modeling obligationes, one fruitful suggestion that presents itself can be rooted in a

\footnotetext{
${ }^{10}$ Yrjönsuuri mentions the possibility of modeling obligationes as games, but he says that "defining the results of the game in any manner appropriate to modern game-theory seem utterly problematic" though despite this "[i]n the following I will keep to the English word game, assuming that the problems pointed out above can just be left unsolved" (Yrjönsuuri 1994, pp. 9-10).
} 
particularly interesting interpretation of obligationes given by King (1991, 2004). In King (2004), King takes his starting point from Spade, who, in Spade (1993), looked to the textual evidence for actual uses of obligationes to understand how they were used by the medievals. While to date there is no historical record for actual obligational disputations, we have many examples of philosophers using obligational techniques as part of their argumentation (King 2004, p. 1). King explains the apparent "content-freeness" of obligational disputations by pointing out that "they operate at a higher level of logical generality than that at which substantive debate occurs. If this is correct, then actual obligational moves-perhaps even recognized as such — are the vehicle whereby real argument takes place" (King 2004, p. 6), and thus obligationes provide a "meta-methodology" for reasoning (King 2004, p. 7).

We use this suggestion as the motivation for our approach to modeling obligationes. An obligatio is essentially a dialogue; and any dialogue can be seen as a game played according to the rules specified by a FDS (Maudet 2003). We believe that viewing obligationes as FDSs, which require that we explicitly specify the logic of argumentation/inference and the models against which the dialogue is to be evaluated, provide a more fruitful approach to modeling obligationes. On this view, Hamblin's modeling approach has the advantage over others proposed in recent literature because it takes the dialogical nature of the disputation seriously. By varying the rules governing the disputation, radically different types of obligationes arise, which result in radically different types of dialogues/disputations. Despite the wide range of difference that can be found, the basic structure of an obligatio remains the same, making the general framework of FDSs an appropriate modeling choice. Specifying obligationes from within the context of FDSs allows us to situate them formally in current research on formal dialogues, which in turn can help to clarify the interpretational question, by helping us understand the possible purposes to which obligationes could be disposed. In particular, we argue that the naturalness of modeling obligationes as dialogue systems supports King's suggestion that obligationes provide agents with a meta-methodology for argumentation. That is, obligationes give frameworks within which dialectical argumentation-dialoguecan take place.

\section{Formal Dialogue Systems}

The standard taxonomy of formal dialogue systems is based on the argumentationbased typology given Walton and Krabbe (1995), who identify six different basic types of dialogues: information seeking, inquiry, persuasion, negotiation, deliberation, and eristic. The division is made on the basis of the preconditions and postconditions that must hold for successful dialogue to take place (Cogan et al. 2006; McBurney and Parsons 2009). Three of these classes, information seeking, inquiry, and persuasion, deal with beliefs and knowledge, and thus are of especial interest, particularly persuasion dialogues, which are " $[\mathrm{t}]$ he only kind of dialogue about $p$ in which one can engage when one knows $p$ " (Cogan et al. 2006, pp. 161162). Walton and Krabbe make no claim as to the comprehensiveness of their classification, and others (Baker 2000; Cogan et al. 2005, 2006; Girle 1996; 
Ravenscroft and Matheson 2002; Sklar and Parsons 2004) have researched types not covered in the Walton and Krabbe typology. In particular, Cogan et al. argue that "there remain several situations in which it seems natural to engage in dialogues, but to which the basic Walton and Krabbe dialogue types do not apply" (Cogan et al. 2006, p. 161). In Cogan et al. (2005, 2006), they take a systematic approach to defining dialogue types on the basis of pre- and post-conditions, and extend Walton and Krabbe's list of belief-based dialogues with four new types, verification and three types of queries. In Sect. 7, we discuss how obligationes fit into these different types. First, we make precise what we mean by a formal dialogue system.

In this section, we follow the presentation of formal dialogue systems given in Prakken (2006), an overview paper which discusses different formal argumentation systems that have been proposed for the analysis of persuasion dialogues and provides a unified approach within which each of these different systems can be modeled. While Prakken focuses on persuasion dialogues, his framework is in fact general enough to handle other types as well (Prakken 2006, pp. 170, 173). Thus, it is appropriate to use it to consider obligationes.

The specification of a formal dialogue system contains the following elements (Prakken 2006, p. 166):

- A topic language $\mathcal{L}_{t}$, closed under classical negation.

- A communication language $\mathcal{L}_{c}$. We denote the set of dialogues, that is, the set sequences of $\mathcal{L}_{c}$, by $M \leq \infty$, and the set of finite sequences of $\mathcal{L}_{c}$ by $M^{<\infty}$. For a dialogue $d=m_{0}, \ldots, m_{n}, \ldots$, the subsequence $m_{0}, \ldots, m_{i}$ is denoted $d_{i}$.

- A dialogue purpose or goal.

- A set $\mathcal{A}$ of agents (participants) and a set $\mathcal{R}$ of roles that the participants can occupy. Each participant $a$ has a (possibly empty) belief base $\Sigma_{a} \subseteq \mathcal{L}_{t}$ and a (possibly empty) commitment set $\mathrm{C}_{a}\left(d_{n}\right) \subseteq \mathcal{L}_{t}$. The belief base may or may not change during the dialogue; the commitment set usually does.

- A context $K \subseteq \mathcal{L}_{t}$, representing the (shared, consistent, and unchanging) knowledge of the agents specified at the outset.

- A logic $L$ for $\mathcal{L}_{t}$.

- A set $E$ of effect rules $\mathrm{C}_{a}\left(d_{n}\right): M^{<\infty} \rightarrow \mathcal{P}\left(\mathcal{L}_{t}\right)$ for $\mathcal{L}_{t}$, specifying how utterances $\varphi \in \mathcal{L}_{c}$ in the dialogue affect the commitment stores of the agents. The effect rules are such that if $d=d^{\prime}$ then $\mathrm{C}_{a}(d, m)=\mathrm{C}_{a}\left(d^{\prime}, m\right)$, that is, the changes in commitments are determined solely by the most recent move in the dialogue along with the commitments at that step.

- A protocol $\mathrm{P}$ for $\mathcal{L}_{c}$, specifying the legal moves of the dialogue, which is a function from the context and a non-empty $D \subseteq M^{<\infty}$ to $\mathcal{P}\left(\mathcal{L}_{c}\right)$, satisfying the requirement that if $d \in D$ and $m \in \mathrm{P}(d)$, then $d, m \in D$. The elements of $D$ are called legal finite dialogues, and $\mathrm{P}(d)$ is the set of moves allowed after move $d$. At any stage, if $\mathrm{P}(d)=\emptyset$, then the dialogue has terminated. A protocol will often be accompanied by a turn-taking function $T: D \rightarrow \mathcal{P}(\mathcal{A})$, which takes a finite dialogue $d_{n}$ and specifies who governs move $m_{n+1}$, and termination conditions, which specify when $\mathrm{P}(d)=\emptyset$.

- A set of outcome rules $O$. 
In this list of components, we can identify the four fundamental building blocks of any formal dialectical system named by Walton: (1) the two participants, called the proponent and the respondent, (2) the types of moves (taking the form of various speech acts) that the two participants are allowed to make, as each takes his or her turn to speak, (3) the sequence of moves, in which the appropriateness of each move depends on the type of preceding move made by the other part, (4) the goal of the dialogue as a whole (Walton 2000, p. 334). In a FDS, we do not restrict ourselves to merely two participants, and of course what we call them is irrelevant. The communication language governs (2), and the protocol specifies (3). The dialogue purpose is (4).

Dialogue systems can be explicitly connected with the games they specify by defining the agents' strategies in the standard game-theoretical way. Formally, a strategy $s_{a}$ for agent $a$ is a function $D_{a} \rightarrow \mathcal{L}_{c}$, where $D_{a} \subseteq D$ is the set of all finite legal dialogues $d_{n}$ in which $T\left(d_{n}\right)=a$. A strategy $s_{a}$ is called winning if in every dialogue where $a$ follows this strategy, he realizes his dialogue goal.

We can identify a number of properties of protocols (Prakken 2006, p. 170):

- A protocol has public semantics iff the set of legal moves is always independent from the agents' belief bases.

- A protocol is context-independent iff the set of legal moves and the outcome is always independent of the context, that is, $\mathrm{P}(K, d)=P(\emptyset, d)$.

- A protocol is fully deterministic iff $\mathrm{P}$ always returns a singleton or the empty set.

- A protocol is unique-move iff the turn shifts after each move; it is multiple-move otherwise.

Protocols which are not fully deterministic are permissive, that is, they specify what moves are legal or allowed for the agent, rather than specify what moves are required. Thus, obligationes are a type of dialogue system where the protocol for the Respondent is fully deterministic; for each proposition the Opponent puts forward, there will be exactly one correct move that the Respondent can make. (In the second example given in Table 2, there is one correct move for the Respondent, but the problem is that he fails to make it when he responded to (2).)

We now show how generically obligationes can be viewed as FDSs; we give precise examples in Sect. 6. In obligationes, there are two designated roles Opp (Opponent) and Res (Respondent) that members of $\mathcal{A}$ can have; those members of $\mathcal{A}$ which do not fill either role are irrelevant for modeling the disputation. We explain below how $\Sigma_{\text {Opp }}, \Sigma_{\text {Res }}, C_{\text {Opp }}, C_{\text {Res }}$, and the context $K$ are generated. In Burley-style positio, the dialogue purpose is consistency: If we take Res's commitment set to be the set of formulas he has conceded along with the negation of those that he's denied over the course of a positio, then the goal for Res is to maintain the consistency of his commitment set, and the goal for Opp is to force Res into contradiction.

In general, the topic language $\mathcal{L}_{t}$ and the communication language $\mathcal{L}_{c}$ are the same. This allows, among other things, the participants in an obligatio to dispute about the allowed moves of the other players. (For example, Opp may ask Res to respond to the claim "You deny $\varphi$ ".) The turn-taking protocol in an obligatio is 
unique-move: $T(\emptyset)=$ Opp, $T\left(d_{n}\right)=$ Opp if $n$ is odd, and $T\left(d_{n}\right)=$ Res if $n$ is even. (Throughout we assume that we label the steps in the sequence from 0 , so in an obligatio it is always Opp that goes first.) The protocol $\mathrm{P}$ will be such that the moves of Opp are not constrained in any way, but Res's moves must be made in reaction to the move of Opp at the previous stage. The same will be true for the effect rules $E$; in a disputation, Opp makes a series of claims or assertions, but these actions have no effect on his commitment store. On the other hand, Res is constrained to be reactive only: He can only concede statements claimed by Opp, concede their negations, or remain ambivalent. Res never asserts any statement of his own devising, he only ever responds to propositions put forward by Opp. Thus, obligationes are essentially asymmetric, in that the rules governing the behavior of the Opp and Res are disjoint, ${ }^{11}$ and so are their actions.

The outcome rules for obligationes are simple: If Res realizes the goal, then he wins. If Opp realizes the goal, then he wins. There is nothing further that hinges upon winning or losing an obligational disputation (except, of course, the individual prestige or embarrassment of the participants!).

Above we noted that in an arbitrary dialogue system, the commitment set of an agent will generally change during the course of the dialogue. It can either strictly grow, so that the agents are only adding new propositions to their commitment-base at each turn, or they can also revise their commitments by rejecting previous commitments in favor of new ones. This latter case arises in ordinary circumstances when agents utilize a form of default reasoning, which is defeasible and nonmonotonic, in that an agent can be forced to accept information which contradicts his previous commitments, requiring that his commitments be revised in order to maintain consistency (cf. Bondarenko et al. (1997); Brewka (2001)). In AI contexts, the ability to simulate non-monotonic reasoning is of great importance; monotonic dialogues and discussions are more commonly found in philosophical contexts. One of the benefits of Prakken's approach to dialogue systems is that it can handle both approaches, merely by the specification of the underlying logic (Prakken 2006, p. 173).

\section{The Underlying Logic}

By specifying the logic $L$ and its underlying models, we are able to explicitly generate $\Sigma_{\mathrm{Opp}}, \Sigma_{\mathrm{Res}}, C_{\mathrm{Opp}}, C_{\mathrm{Res}}$, and $K$ satisfying desired properties. In our approach to modeling obligationes as FDSs, the underlying logic is a variant of multi-agent Dynamic Epistemic Logic (DEL, van Ditmarsch et al. 2007). This logic is monotonic and not argument based. An epistemic logic (EL) is an extension of propositional logic with a family of modal operators $K_{a}$ for $a \in \mathcal{A}$. We are interested

\footnotetext{
${ }^{11}$ In fact, in most texts, no rules for Opp are given. One exception is the early text Tractatus Emmeranus de Rijk (1974), which gives some rules (better thought of as guidelines, or strategic advice) to the Opponent.
} 
in a particular extension of standard epistemic logic, namely, epistemic logic with common knowledge, which has a further family of operators $C_{G}$, for $G \subseteq \mathcal{A}$. For a set $\Phi_{0}$ of propositional letters and set $\mathcal{A}$ of agents, the set $\Phi_{\mathrm{EL}}^{\mathcal{A}}$ of well-formed formulas of EL is defined by:

$$
\varphi:=p \in \Phi_{0}|\neg \varphi| \varphi \vee \varphi\left|K_{a} \varphi: a \in \mathcal{A}\right| C_{G} \varphi: G \subseteq \mathcal{A}
$$

$K_{a} \varphi$ is read 'agent $a$ knows that $\varphi$ '. $C_{G} \varphi$ is read 'it is common knowledge amongst the group of agents $G$ that $\varphi$ '. $C_{G}$ is used to give an explicit representation of the knowledge of the two agents at the beginning of the disputation.

The models for epistemic logic are Kripke models. A Kripke model $\mathfrak{M}=\left\langle W, w^{*},\left\{\sim_{a}: a \in \mathcal{A}\right\}, V\right\rangle$ is an epistemic model if

- $\quad W$ is a set (of possible worlds), with $w^{*} \in W$ a designated point (representing the actual world).

- $\left\{\sim_{a}: a \in \mathcal{A}\right\}$ is a family of equivalence relations on $W$, one for each member of $\mathcal{A}$. The relation $w \sim{ }_{a} w^{\prime}$ is interpreted as ' $w$ and $w^{\prime}$ are epistemically equivalent for agent $a^{\prime} . \sim_{G}: G \subseteq \mathcal{A}$ is defined as the reflexive and transitive closure of $\bigcup_{a \in G}\{\sim a\}$.

- $V: \Phi_{0} \rightarrow 2^{W}$ is a valuation function associating atomic propositions with subsets of $W$. For $p \in \Phi_{0}$, if $w \in V(p)$, we say that ' $p$ is true at $w$ '.

The semantics for the propositional connectives and the epistemic operators are as follows:
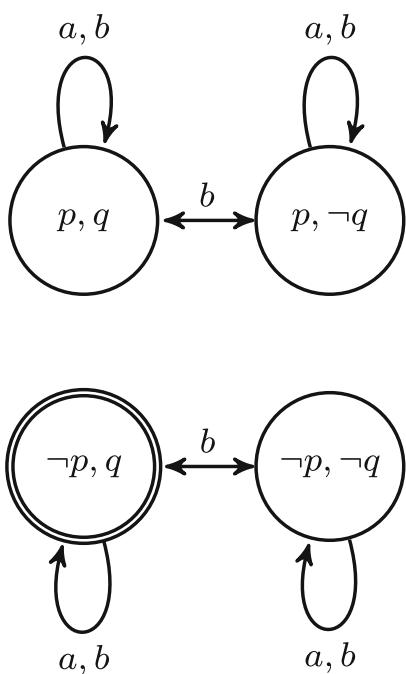

Fig. 1 An example Kripke model 


$\begin{array}{lll}\mathfrak{M}, w \vDash p & \text { iff } & w \in V(p) \\ \mathfrak{M}, w \models \neg \varphi & \text { iff } & \mathfrak{M}, w \nvdash \varphi \\ \mathfrak{M}, w \vDash \varphi \wedge \psi & \text { iff } & \mathfrak{M}, w \vDash \varphi \text { and } \mathfrak{M}, w \vDash \psi \\ \mathfrak{M}, w \models \varphi \vee \psi & \text { iff } & \mathfrak{M}, w \vDash \varphi \text { or } \mathfrak{M}, w \vDash \psi \\ \mathfrak{M}, w \vDash \varphi \rightarrow \psi & \text { iff } & \mathfrak{M}, w \vDash \varphi \text { implies } \mathfrak{M}, w \vDash \psi \\ \mathfrak{M}, w \vDash K_{a} \varphi & \text { iff } & \forall w^{\prime}\left(\text { if }\left\langle w, w^{\prime}\right\rangle \in \sim{ }_{a} \text { then } \mathfrak{M}^{E}, w^{\prime} \vDash \varphi\right) \\ \mathfrak{M}, w \vDash C_{G} \varphi & \text { iff } & \forall w^{\prime}\left(\text { if }\left\langle w, w^{\prime}\right\rangle \in \sim_{G} \text { then } \mathfrak{M}^{E}, w^{\prime} \vDash \varphi\right)\end{array}$

An example Kripke model is given in Fig. 1. There are four worlds representing the four possible combinations of truth values of atoms $p$ and $q$, and two agents, $a$ and $b$. The relationship of epistemic equivalence between worlds is indicated by the arrows, labeled with the agents. The actual world is the world where $\neg p, q$ holds (indicated by the double ring), and agent $b$ knows that this is the actual world. Agent $a$, on the other hand, knows that $p$ is false, but is uncertain about the truth of $q$, and even if it were the case that $p$ were true, agent $b$ would still be uncertain about $q$.

Epistemic logic models cover the knowledge of the agents; to model their actions, we add dynamics, via Propositional Dynamic Logic (PDL, Harel et al. (2002)). PDL is an extension of propositional logic by a family of modal operators $[\alpha]$ for $\alpha \in \Pi$, a set of programmes (or more generally, a set of actions or events). The language of PDL is two-sorted, with a set $\Phi_{0}$ of atoms and a set $\Pi_{0}$ of atomic actions. We do not need the full expressivity of PDL to model obligationes, so we introduce only the fragment we require. We let $\Pi_{0}=\emptyset$, and the sets $\Phi_{\mathrm{Ob}}$ and $\Pi_{\mathrm{Ob}}$ of complex well-formed formulas and programmes are defined by mutual induction:

$$
\begin{aligned}
\varphi & :=\varphi \in \Phi_{\mathrm{EL}}^{\mathcal{A}} \mid[\alpha] \varphi: \alpha \in \Pi_{\mathrm{Ob}} \\
\alpha & :=\varphi ?: \varphi \in \Phi_{\mathrm{EL}}^{\mathcal{A}}
\end{aligned}
$$

The programme $\varphi$ ? is to be interpreted as a test operator, which tests for the truth of $\varphi$. Note that the only programmes that we allow are testing of formulas which do not themselves contain any programmes. The semantics for the new $[\varphi$ ?] operator are given in terms of model restriction. Let $\mathfrak{M}\left\lceil\varphi:=\left\langle W^{\mathfrak{M}, \varphi},\{\underset{a}{\mathfrak{M}, \varphi}: a \in \mathcal{A}\}\right.\right.$, $\left.V^{\mathfrak{M}, \varphi}\right\rangle$, where $W^{\mathfrak{M}, \varphi}:=\{w \in W: \mathfrak{M}, w \vDash \varphi\}$, and the relations and valuation functions are just restrictions of the originals. For a set of ordered propositions $\Gamma_{n}$, let $\mathfrak{M}\left\lceil\Gamma_{n}=\mathfrak{M}\left\lceil\gamma_{0} \uparrow \cdots \uparrow \gamma_{n}\right.\right.$, that is, $\mathfrak{M} \uparrow \Gamma_{n}$ is the result of the sequential restriction of $\mathfrak{M}$ by the elements of $\Gamma_{n}$. Then:

$$
\mathfrak{M}, w \vDash[\varphi ?] \psi \quad \text { iff } \quad \forall v \in \mathfrak{M}\lceil\varphi, v \vDash \psi
$$

We give examples of how these test operators and model restrictions work at the end of Sect. 6.

One advantage of using an epistemic logic for our disputation logic is that it allows us to model the epistemic bases of the agents, and the context of the disputation, explicitly (for a fuller discussion of the advantages, which includes the ability to use this framework to model different types of obligationes beyond just the one considered here, see Uckelman (2011b)). While above we defined the concept of a "belief base" in a dialogue, in the context of obligationes it is the 
agent's knowledge, not his beliefs, that is important. Given an epistemic model $\mathfrak{M}$, the knowledge bases of Opp and Res are defined as follows:

$$
\begin{aligned}
& \Sigma_{\text {Opp }}^{\mathfrak{M}}:=\left\{\varphi: \mathfrak{M}, w^{*} \vDash K_{\text {Opp }} \varphi\right\} \\
& \Sigma_{\text {Res }}^{\mathfrak{M}}:=\left\{\varphi: \mathfrak{M}, w^{*} \vDash K_{\text {Res }} \varphi\right\}
\end{aligned}
$$

That is, the knowledge base of the two players is simply the propositions he knows in the actual world.

In an arbitrary model $\mathfrak{M}$, the set of propositions which are common knowledge amongst a group of agents is not explicitly specified. In an obligatio, the set of common knowledge, against which the truth of irrelevant propositions is evaluated, is likewise often left implicit. In some cases, before the obligatio begins, a casus is introduced. ${ }^{12}$ A casus is a hypothesis about how the world is, or extra information about how the positum should be analyzed (Yrjönsuuri 1993). A common example of a positum introduced with a casus (understood in the first sense) is "In truth Socrates is black. It is posited that Socrates is white." The first sentence is the casus; it tells the participants not only that Socrates is black, but also that he exists, and is colored, all of which facts the Respondent must take into account when responding to the Opponent's posita. Thus, in the first sense, the casus can be understood as a set of literals expressing the explicit common knowledge at the start of the dialogue, so the casus can be implemented by a restriction on $V$.

Definition 1 (Casus) Let $\operatorname{Lit}_{\Phi_{0}}$ be the set of literals formed from $\Phi_{0}$, and $K \subseteq$ Lit $_{\Phi_{0}}$ be the casus. Then $\mathfrak{M}$ models the casus if there is a $P_{c} \subseteq P$ of $W$ with $w^{*} \in P_{c}$, such that if $w \sim \operatorname{Res} w^{*}$, then $w \in P_{c}$, if $v \sim \mathrm{Opp}^{*} w^{*}$, then $v \in P_{c}$, and for all $w, v \in$ $P_{c}, w \sim \operatorname{Res} v$ and $w \sim \operatorname{Opp} v$; and for every positive literal $p \in K$ and every $w \in$ $P_{c}, w \in V(p)$, and for every negative literal $\neg q \in K$ and every $w \in P_{c}, w \notin V(q)$.

Unlike contexts in dialogue systems, it is not assumed that the casus of an obligatio is consistent, but if it is not, then Res should not accept the positum, since Opp could easily force him into conceding a contradiction. However, if the casus is consistent, we can easily show that if $\mathfrak{M}$ models a casus $K$, then for every $\varphi \in K, \mathfrak{M} \vDash C_{\{\text {Opp,Res }\}} \varphi$, and so $K \subseteq \Sigma_{\text {Opp }}^{\mathfrak{M}}$ and $K \subseteq \Sigma_{\text {Res }}^{\mathfrak{M}}$.

\section{Protocols, Effect Rules, and Outcomes}

Different types of obligationes can be modeled by changing the protocols, effect rules, and outcome conditions. First, we specify the general properties shared by all

\footnotetext{
12 The use of the casus is more common in post-Burley treatises; Burley only uses the term in an off-hand fashion; in one example he says, "Let it be the case that Socrates is black, and let it be put forward that Socrates is white. However the case does not constrain [the Respond] but certifies, and because it is possible to be certain of the truth of one of the opposites and to sustain the other as true, it is possible to admit a positio in which it is put forward that Socrates is white, when previously it was said in the truth of things, 'Socrates is black' " "'Sit Socrates niger, et ponatur Socratem esse album. Sed casus non obligat sed certificat, et quia possum esse certus de veritate unius oppositorum et sustinere reliquum pro vero, potest admitti positio quae ponit Socratem esse album, prius dicto in rei veritate: 'Socrates est niger'" (Burley 1963, §C.1)).
} 
obligationes. We identify our set of agents with their roles, i.e., our set of agents is $\mathcal{A}=\{\mathrm{Opp}, \mathrm{Res}\}$, and our topic language and commitment language is the language of Dynamic Epistemic Logic $\mathcal{L}_{\text {DEL }}$ introduced in the previous section. Let $\alpha$ be a designated formula representing "cedat tempus". We can identify two types of protocols used in obligationes. The first type of protocol is uniform throughout all different systems; the second varies from author to author and type to type. The uniform protocol $\mathrm{P}_{u}$ is invariant over all contexts and is defined for a finite dialogue $d_{n}$ :

$$
\begin{array}{ll} 
& \mathrm{P}_{u}(\emptyset)=\mathcal{L}_{c} \\
\text { if } m_{n}=\alpha & \mathrm{P}_{u}\left(d_{n}\right)=\emptyset \\
\text { otherwise, if } n \text { is odd, } & \mathrm{P}_{u}\left(d_{n}\right)=\mathcal{L}_{c} \\
\text { and if } n \text { is even, } & \mathrm{P}_{u}\left(d_{n}\right)=\left\{\left[m_{n} ?\right] \top,\left[\neg m_{n} ?\right] \top,[\top ?] \top\right\}
\end{array}
$$

That is, if it is Opp's turn, he is allowed to assert any statement in the communication language (we allow repetitions). If it is Res's turn, he must either concede, deny, or doubt Opp's statement from the previous round. And if "cedat tempus" has been called, the dialogue ends and there are no more legal moves available. Since $m_{n}$, the move of Opp, will always be a statement in the communication language $\mathcal{L}_{c}$, and the communication language allows for the embeddings of the test programme, this protocol is well-defined. For ease of future reference, we introduce meta-names for the actions of Res : concede: $\varphi:=\left[\varphi_{n} ?\right] \top$, deny: $\varphi:=\left[\neg \varphi_{n} ?\right] \top$, and doubt: $\varphi:=[\top ?] \top$. The actions of concession and denial are to be understood as asking (i.e., testing) whether $\varphi$ or $\neg \varphi$ are consistent. The last action is equivalent to saying "I don't know"; [T?] $\top$ will always be valid, in any model, since $\top$ is necessarily true.

This protocol has public semantics and is context-independent, but it is not fully deterministic, since whenever it is Res's turn, he has a choice of actions.

The rules governing the commitment sets $\mathrm{C}_{\mathrm{Opp}}$ and $\mathrm{C}_{\mathrm{Res}}$ are defined as follows:

$$
\begin{array}{ll}
\text { for all } n & \mathrm{C}_{\text {Opp }}\left(d_{n}\right)=\emptyset \\
\text { if } n \text { is even } & \mathrm{C}_{\operatorname{Res}}\left(d_{n}\right)=\mathrm{C}_{\operatorname{Res}}\left(d_{n-1}\right) \\
\text { if } n \text { is odd } & \mathrm{C}_{\operatorname{Res}}\left(d_{n}\right)=\mathrm{C}_{\operatorname{Res}}\left(d_{n-1}\right) \cup\left\{m_{n}\right\}
\end{array}
$$

That is, Opp has no commitments, Opp's moves do not change Res's commitments, and Res's commitment store strictly grows on the basis of his actions, and thus obligational dialogues are monotonic. As above, since $\mathcal{L}_{c}$ and $\mathcal{L}_{t}$ coincide, the final clause of the definition is well-defined. Note that in general, $C_{\text {Res }}$ and $\Sigma_{\text {Res }}$ will be disjoint, and similarly for $\mathrm{C}_{\text {Res }}$ and $K$ (contra, e.g., (Parsons et al. 2002, §3), where "an agent's commitment store is just a subset of its knowledge base").

The general protocol defined above specifies what the possible moves of Res are. In an obligatio, however, we want to say more than what moves are allowed, we also want to specify a set of possible moves which are in fact required, since in an obligational disputation Res is under obligation to respond to Opp in certain ways. This is done by specifying a more refined protocol. Such a protocol, because it makes reference to the agents' knowledge bases, will always be defined with respect to a particular DEL model $\mathfrak{M}$. We give as an example Burley's protocol $\mathrm{P}^{\mathrm{Bur}}$ for positio, introduced in 
Sect. 2. Let $\Gamma_{n}$ be the sequence of Res's move in a dialogue $d_{n}$. For a DEL model $\mathfrak{M}$ and context $K, \mathrm{P}^{\mathrm{Bur}}(K, \emptyset)=P_{u}(\emptyset)$ and if $n$ is odd, $\mathrm{P}^{\mathrm{Bur}}\left(K, d_{n}\right)=P_{u}\left(d_{n}\right)$. For $n$ even,

- For $d_{0}=m_{0}=$ the positum,

$$
\mathrm{P}^{\mathrm{Bur}}\left(K, d_{0}\right)= \begin{cases}\text { concede: } m_{0} & \text { iff } \exists w \in W, \mathfrak{M}, w \vDash m_{0} \\ \text { deny: } m_{0} & \text { iff } \forall w \in W, \mathfrak{M}, w \not \models m_{0}\end{cases}
$$

- For $d_{n}, n>0$ :

$$
\begin{array}{ll}
\text { If } \mathfrak{M} \uparrow \Gamma_{n} \vDash m_{n}: & \mathrm{P}^{\mathrm{Bur}}\left(K, d_{n}\right)=\text { concede: } m_{n} \\
\text { If } \mathfrak{M} \uparrow \Gamma_{n} \vDash \neg m_{n}: & \mathrm{P}^{\mathrm{Bur}}\left(K, d_{n}\right)=\text { deny: } m_{n} \\
\text { Otherwise: } & \\
\text { If } \mathfrak{M}, w^{*} \vDash K_{\text {Res }} m_{n}: & \mathrm{P}^{\mathrm{Bur}}\left(K, d_{n}\right)=\text { concede: } m_{n} \\
\text { If } \mathfrak{M}, w^{*} \models K_{\text {Res }} \neg m_{n}: & \mathrm{P}^{\mathrm{Bur}}\left(K, d_{n}\right)=\text { deny: } m_{n} \\
\text { If } \mathfrak{M}, w^{*} \models \neg\left(K_{\operatorname{Res}} m_{n} \vee K_{\operatorname{Res}} \neg m_{n}\right): & \mathrm{P}^{\mathrm{Bur}}\left(K, d_{n}\right)=\text { doubt }: m_{n}
\end{array}
$$

Finally, we define two outcome rules for Burley-style positio, governing who wins. Generally speaking, Opp wins if he can force Res into inconsistency, and Res wins otherwise. Since any individual obligatio $=d_{n}$ for some finite $n$, we can define a weak notion of "local" winning: If $m_{n}=\alpha$, then Opp wins if $\mathfrak{M}\left\lceil\Gamma_{n}=\left\langle\emptyset,\left\{\sim{ }_{a}^{\mathfrak{M}, \Gamma_{n}}: a \in A\right\}, V^{\mathfrak{M}, \Gamma_{n}}\right\rangle\right.$ and Res wins otherwise. But even though individual obligationes are finite, they are all potentially infinite. This view gives rise to a "global" winning condition: Opp wins if there is some $n$ such that $\mathfrak{M}\left\lceil\Gamma_{n}=\left\langle\emptyset,\left\{\sim_{a}^{\mathfrak{M}, \Gamma_{n}}: a \in A\right\}, V^{\mathfrak{M}, \Gamma_{n}}\right\rangle\right.$. Res wins otherwise. In both cases, the only time $W$ will be empty is when $\mathrm{C}_{\text {Res }} \vDash \varphi \wedge \neg \varphi$, that is, over the course of the disputation Res has conceded an inconsistent set, and has thus "responded badly". Thus, protocol $\mathrm{P}^{\mathrm{Bur}}$ ensures the dialogical consistency of Res (cf. Prakken (2006, p. 171) and Dutilh Novaes (2007, ch. 3)).

There are also two ways that "responded badly" can be explicated, a broadgrained way and a fine-grained way. On the broad-grained view, we are only interested in whether Opp or Res has locally won, that is, whether Opp has been able to force Res to concede a contradiction, or whether Res has remained consistent in his answers. This is the view generally considered by medieval authors. However, we may also be interested in a more fine-grained notion of

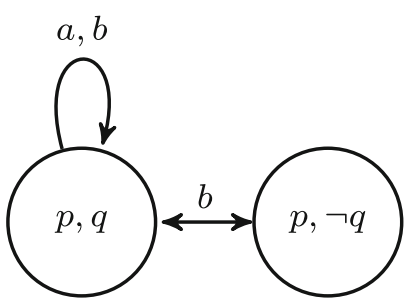

Fig. $2 \mathfrak{M}\left\lceil d_{1}\right.$ 


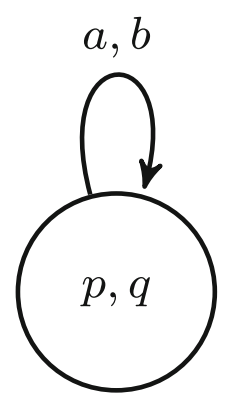

Fig. $3 \mathfrak{M}\left\lceil d_{1}, d_{3}\right.$

correct response, namely, knowing whether Res has actually followed the rules he was constrained to follow, and not just that he succeeded in maintaining a contradiction-free stance.

Before we discuss some interesting properties of protocols like the ones introduced here, we return to the example in Table 1 , using the model $\mathfrak{M}$ given in Fig. 1 with $\varphi=p$ and $\psi=q$. This model is such that there is a $w \in W, \mathfrak{M}, w \vDash \varphi \wedge \psi$, and it models the casus $K=\{\neg \varphi\}$. The first move is Opp's, and he asserts the positum $\varphi:=d_{0}$. According to $\mathrm{P}^{\mathrm{Bur}}\left(d_{0}\right)$, Res should concede: $\varphi:=d_{1}$ (Fig. 2). The protocol now allows Opp to make any assertion he likes, so he asserts $\neg \varphi \vee \psi:=d_{2}$. Now, by the argument given above, either $\mathfrak{M}\left\lceil d_{1} \vDash d_{2}\right.$, or, if not, then $\mathfrak{M}, w^{*} \vDash K_{\text {Res }} d_{2}$. By the protocol, in both cases, Res should concede and $d_{3}=$ concede: $d_{2}$. Then Opp asserts $d_{4}=\psi$, and since $\mathfrak{M}\left\lceil d_{1}, d_{3}=\psi\right.$, (Fig. 3) Res is again required to respond with concede: $d_{4}:=d_{5}$. Then, Opp calls "cedat tempus", and $d_{6}=\alpha$, and, by the general protocol $\mathrm{P}_{u}$ there are no more legal moves and the dialogue ends. Because the final model is nonempty, Res has succeeded in maintaining consistency, and hence he wins.

\section{Discussion}

\subsection{The Protocol}

The protocol $\mathrm{P}^{\mathrm{Bur}}$ defined above is semi-public, as it depends on Res's knowledge, but does not depend on Opp's; context-dependent; and fully deterministic. It also meets all but four of the 13 desiderata for agent argumentation protocols given in McBurney et al. (2002). There McBurney et al. consider dialogue protocols from the point of view of design, and identify 13 desiderata that a designer might want to incorporate. These are:

- stated dialogue purpose A system should have one or more publicly stated purpose, and its structure should facilitate its achievement. ${ }^{13}$

\footnotetext{
13 Cf. "The sequence of moves should ideally move towards the fulfillment of the goal as the dialogue proceeds" (Walton 2000, p. 334).
} 
- diversity of individual purposes Participants should be allowed to have their own (distinct) purposes, consistent with the stated dialogue purpose.

- inclusiveness Any qualified participant who wants to participate can.

- transparency Participants should know the rules and structure of the system prior to its commencement.

- fairness Either all participants should be treated equally, or asymmetries in treatment should be explicit.

- clarity of argumentation theory The dialectical system should conform to a stated theory of argumentation.

- separation of syntax and semantics The syntax and the semantics of the system should be defined separately.

- rule-consistency The rules and locutions should not lead to deadlocks or to infinite cycles of repeated locutions.

- encouragement of resolution Resolution should not be precluded by the rules.

- discouragement of disruption The rules should discourage disruptive behavior, such as repeating the same locution repeatedly.

- enablement of self-transformation Participants should be able to change their preferences, knowledge, degrees of belief, etc.

- system simplicity The locutions and rules of the system should be as simple as possible, consistent with the other desiderata.

- computational simplicity The system should minimize computational demands on the participants.

In the FDS constructed from Burley's obligational theory, the dialogue purpose is stated explicitly (cf. Sect. 2). The purposes of Res and Opp are distinct, and anyone who is qualified to participate in an obligatio as either Res or Opp may. The rules are agreed upon in advance, and the asymmetry between Res and Opp is explicit. The system conforms to a stated theory of argumentation, namely Burley's rules for positio. The rules do not preclude resolution, and it is also quite simple, statable in a case-based structure with minimal cases to consider. Further, to the extent that the protocol is rule-consistent (which we discuss below), it discourages disruption in that Res is never able to continuously repeat the same locution, at least, not without responding badly.

The issue of self-transformation enablement requires further comment. McBurney et al. further specify that self-transformation requires that "participants should have the right to retract commitments made earlier in the same dialogue" for otherwise "in such circumstances, there would be no point for the agents to engage in dialogue" (McBurney et al. 2002, p. 403). We disagree with this assessment; the obligationes framework provides a counterexample, a type of dialogue which is worthwhile engaging in but in which the agents' commitments are monotonic. Obligationes satisfy the less restrictive view of self-transformation, given that the commitment sets and responses of Res are flexible and can change over the course of the disputation.

For the remaining desiderata, because the topic language and the communication language coincide, it is not clear to what extent obligationes satisfy the requirement of the separation of syntax and semantics. It does not satisfy rule-consistency or 
discouragement of disruption, because Opp can continually put forward the same proposition; however, while the protocol allows Opp to act in such a fashion, there are pragmatic reasons why he will generally not do so. If Res responds well (that is, follows the protocol), the only time he will change his response to a proposition $\varphi$ is to move from doubt to either concede or deny. If he has responded badly at some point, then Opp may by repeating a locution be able to force Res into losing, in which case there will be no point in Opp repeating that proposition again, as he should instead call "Cedat tempus". Whether the final criterion is satisfied is uncertain: As we note in the final section, the computational complexity of certain decision problems that can be extracted from this protocol is not yet known. Regardless, the protocol scores quite highly-as well, or better, than the protocols analysed in McBurney et al. (2002). It should also be clear that this high score is not specific to Burley's protocol: Any other FDS protocol developed from a different medieval theory would score similarly high.

\subsection{Comparison with Other Dialogue Systems}

We discussed Walton and Krabbe's taxonomy of dialogue types, and Cogan et al.'s extensions, in Sect. 4 before introducing Prakken's framework. Prakken himself distinguishes two types of persuasion dialogues, those that are for pure persuasion and those for conflict resolution (Prakken 2006, p. 169). In his terminology, a dialogue system $S$ is for pure persuasion iff for any terminated dialogue $d$, agent $a$ is a winner for topic $t\left(a \in w_{t}(d)\right)$ iff either $a$ is a proponent of $t$ and $t \in \mathrm{C}_{a^{\prime}}(d)$ for all $a^{\prime}$ who are either proponents or opponents of $t$, or $a$ is an opponent of $t$ and $t \notin \mathrm{C}_{a^{\prime}}(d)$ for all $a^{\prime}$ who are either proponents or opponents of $t$. A dialogue system $S$ is for conflict resolution otherwise.

Where do obligationes fit in these schemes? Prakken says that frameworks for persuasion dialogues can be found in the Middle Ages (Prakken 2006, p. 163, citing Angelelli (1970)). Angelelli's discussion of medieval disputations in Angelelli (1970) focuses on obligationes, but nowhere does he make any mention of persuasion. If obligationes are a type of persuasion dialogue, then because Opp's commitment store is always empty in an obligatio, obligationes would be classified, on Prakken's distinction, as conflict resolution dialogues instead of pure persuasion dialogues. However, given the stated goal of obligationes and the fact that the players agree on the truth value of the proposition in question at the start of the dialogue, this classification does not seem appropriate.

Despite its breadth, the decempartite division of Cogan et al. (2006), incorporating Walton and Krabbe's typology, also does not accommodate obligationes. Because they are about the consistency of a formula, obligationes are not negotiation or deliberation dialogues. Because the truth value of the proposition in dispute is known to both, and the Opponent is not trying to persuade the Respondent of anything, they are not information-seeking, inquiry, or pure persuasion dialogues. Since they are not pugilistic in nature, they are not eristic dialogues. Nor are they 
any of the four new kinds introduced in Cogan et al. (2006), since those types require as well that at least one party not know the truth-value of the proposition. ${ }^{14}$

Obligationes are somewhat similar to the 'elicit-inform' dialogue game of Matheson and Ravenscroft (2001); Ravenscroft and Matheson (2002). These dialogues were developed by Ravenscroft and Matheson in the context of collaborative e-learning, where the dialogues are between a tutor system and a student. The asymmetry between the players is similar to the asymmetry between the Opponent and the Respondent. In an elicit-inform dialogue, the student is questioned by the tutorial system, and “after reasoning about the learner's contributions, the tutor system either sanctions their explanations by informing them they were correct, or points out that they were 'incorrect' and so informs them of a consistent, or 'correct' answer" (Ravenscroft and Matheson 2002, p. 96). This is very similar to the behavior of the Opponent when he calls Cedat tempus and evaluates the actions of the Respondent to determine whether he has responded well or badly. However, as elicit-inform dialogues have as their goal the persuasion of the student to adopt a certain belief, they are not a perfect match for obligationes, since persuasion is not at issue in obligational dialogues.

Instead of trying to shoehorn obligationes into a type of dialogue system already introduced in the literature, we think it is more interesting, and highlights the unique nature of obligational reasoning better, to consider them as introducing a new type of dialogue into the typology. Thus, one of the contributions of the current paper is the introduction of a new type of inter-agent dialogue, for checking the feasibility of a set of propositions. Proving that a proposition or a set of propositions is feasiblecan be consistently maintained-has various applications in constraint and allocation satisfaction, where a consistent solution meeting certain requirements has to be found. Thus, the new dialogue type that we have introduced may turn out to have useful applications beyond the context of obligationes, though we do not investigate this potential extension further here.

\subsection{The Role of (Dialectical) Obligations}

One final point of interest, in comparing protocols based on obligationes to other dialogue protocols, is the role of obligations (in the ordinary sense of the term) in the dialogues. Generally, in a FDS, "[s]trictly speaking the only dialectical obligation that a participant has is making an allowed move when it is one's turn" (Prakken 2006, p. 170). In an obligatio, however, Res has a two-tiered obligation: $\mathrm{He}$ is required to follow both the uniform protocol $\mathrm{P}_{u}$, and the appropriate specific

\footnotetext{
${ }^{14}$ However, were one to shift the basis on which dialogues are classified from the initial sentence(s) being disputed to the overall goal of the dialogue, then it could be argued that obligationes are a type of examination dialogue because the Respondent's ability to follow the rules is being examined—a similar suggestion is considered in the next paragraph — and thus, depending on the specific type of examination, fall under either the inquiry or persuasion type. On such a view, it would make sense to understand an obligational disputation as being one where the Respondent's ability to follow the rules is being examined, and thus it is either an inquiry into his abilities, or, if the Respondent and the Opponent differ in their views of Respondent's abilities, a persuasion by one player of the other. I'm grateful to one of the anonymous referees for this suggestion.
} 
protocol for the type of obligatio he is in. ${ }^{15}$ It is possible for Res to violate his obligation to follow the second protocol (in which case he loses), but if he does not follow the uniform protocol than no disputation even takes place. This two-tiered nature of the obligation of the Res helps us understand King's analysis of obligationes as a meta-methodology of argumentation. The specific protocol is the methodology - it tells Res how to respond within a particular disputation-while the general protocol constrains the types of specific protocols that are allowed, and hence can be understood as a meta-methodology (a higher order method).

Furthermore, this two-tiered approach allows us to evaluate an obligatio at both the descriptive and the formal level. Walton, following Hamblin, distinguishes between the descriptive and the formal study of dialogue, and says that:

The descriptive study of dialogue is concerned with actual conversational exchanges like parliamentary debates, legal cross-examinations, and so forth. The formal study of dialogue "consists in the setting up of simple systems of precise but not necessarily realistic rules, and the plotting out of the properties of the dialogues that might be played out in accordance with them" (Hamblin 1970, p. 256) (Walton 2000, pp. 333-334).

At the descriptive level, we can study any obligational disputation from the point of view of the uniform protocol; at the formal level, we can then evaluate such disputations with respect to the rules the Respondent was required to follow, to determine whether he has met his dialectical obligations or not. This distinction parallels with the notion of the intrinsic and extrinsic goal of a dialogue discussed by Gabbay and Woods. These distinctions are useful since they allow that a dialogue "might fail to achieve the specific goal of a dialogue of a certain type without ceasing to be a dialogue of that type" (Gabbay and Woods 2001, p. 162).

Additionally, in an obligatio there is no connection between an agent's commitment store and his assertions; Opp has no commitments, even though all he makes are assertions, and Res makes no assertions, but his commitments are generated from his concessions and denials of Opp's assertions. This is a significant difference from standard commitment rules such as the one discussed by Prakken in his Paul and Olga example (Prakken 2006, p. 169). He says "As for commitment rules, the following ones seem to be uncontroversial and can be found throughout the literature:

- If $s(m)=\operatorname{claim}(\varphi)$ then $C_{s}(d, m)=C_{s}(d) \cup\{\varphi\} \ldots$ "

That is, an agent's discursive commitments are generated from his assertions. A similar position is advocated when he says elsewhere that "Commitments are typically incurred by making or conceding claims and stating" (Prakken 2005, p. 1017).

\footnotetext{
15 The obligation to follow the uniform protocol is what Walton and Krabbe term an 'action commitment': an obligation to execute a particular course of action, given the action(s) of the other player(s) (Walton and Krabbe 1995, ch. 1).
} 


\section{Conclusion}

We have now seen how at least one type of medieval obligational theory can be interpreted as giving rise to a formal dialogue system; it is straightforward to extend this analysis not just to theories of positio outlined by other medieval authors but also other types of obligationes, such as dubitatio, by varying the underlying logic. ${ }^{16}$ The result of such an analysis shows that just as a particular dialogue can be viewed as a game played according to a set of rules specified by a FDS (cf. Sect. 3), so too obligationes can be naturally understood as giving the participants a methodology of argumentation or reasoning to follow. By specifying the protocols and rules of a FDS, a particular obligational theory gives participants a framework within which to do philosophical analysis. This provides formal support for King's interpretation of obligationes as functioning at the meta-level, rather than at the content level, and also shows, quite clearly, that Walton's conclusion, that "after the Greeks... the conversational model of argumentation faded into the background" (Walton 2000, p. 328), is false. We close our paper by pointing towards questions that we hope to answer in future work.

In Sect. 6 we distinguished two ways "responds badly" or "responds well" can be explicated. Both of these give rise to decision problems whose complexity we intend to investigate in future work. In the first, the local winning outcome conditions corresponds to the decision problem ResPONDS-Well $\left(d_{n}, \mathfrak{M}\right)$ defined as follows: Given a finite obligatio $d_{n}$ and model $\mathfrak{M}$, check whether $\mathfrak{M}\left\lceil\Gamma_{n}=\left\langle\emptyset,\left\{\sim_{a}^{\mathfrak{M}, \Gamma_{n}}: a \in A\right\}, V^{\mathfrak{M}, \Gamma_{n}}\right\rangle\right.$, that is, whether Opp has locally won. The answer is not necessarily a straightforward adaptation of complexity results for model-checking in Public Announcement Logic (PAL, (van Ditmarsch et al. 2007, ch. 4)), since in PAL, attention is restricted to announcements which are truthful, and the introduction of announcements which may be false, but which are nonetheless effective (in that they reduce the model), is not generally considered.

The fine-grained view asks whether, at each step $n$, Res has followed the specific protocol he was obligated to follow. It is possible for Res to have responded correctly in the sense of winning locally on the broad-grained view, but to still have not followed the rules correctly, by choosing the wrong response for irrelevant propositions, for example, by conceding an irrelevant proposition known to be false. In this case, the decision problem Rule-Following $\left(d_{n}, \mathrm{P}, \mathfrak{M}\right)$ is defined as follows: Given a finite obligatio $d_{n}$, protocol $\mathrm{P}$, and model $\mathfrak{M}$ check whether the construction of $\Gamma_{n}$ satisfies the conditions of $\mathrm{P}$. It may be possible to extend the complexity results of Parsons et al. (2002, 2003a, b), though it is not prima facie clear how this would be done. In particular, the results in (Parsons et al. 2003b, §7) are for argumentation-based logics, which DEL is not, and the results in (Parsons et al. 2002 , §5-6) are for protocols where repetition is not allowed.

Acknowledgments This paper is an extended version of Uckelman (2011c). Research for this paper was funded by the NWO project "Dialogical Foundations of Semantics" (DiFoS) in the ESF EuroCoRes programme LogICCC (LogICCC-FP004; DN 231-80-002; CN 2008/08314/GW). The author would like to thank both the anonymous referees and participants of the workshop Computational Models of Natural

16 The case of dubitatio is considered in Uckelman (2011a). 
Argument X, August 2010, for their meticulous and useful comments on an earlier version of this paper, and also the two anonymous referees who provided further detailed and helpful comments on this extended version.

Open Access This article is distributed under the terms of the Creative Commons Attribution License which permits any use, distribution, and reproduction in any medium, provided the original author(s) and the source are credited.

\section{References}

Angelelli, I. 1970. Techniques of disputation in the history of logic. Journal of Philosophy 67(20): $800-815$.

Baker, M. 2000. The role and models in artificial intelligence and educational research: A prospective view. International Journal of Artificial Intelligence in Education 11: 122-143.

Boehner, P. 1952. Medieval logic: An outline of its development from 1250 to c. 1400. Manchester: Manchester University Press.

Bondarenko, A., P.M. Dung, R.A. Kowalski, and F. Toni. 1997. An abstract, argumentation-theoretic approach to default reasoning. Artificial Intelligence 93: 63-101.

Braakhuis, H. 1998. Obligations in early 13th century Paris: The obligationes of Nicholas of Paris (?). Vivarium 36(2): 152-233.

Brewka, G. 2001. Dynamic argument systems: A formal model of argumentation processes based on situation calculus. Journal of Logic and Computation 11(2): 257-282.

Burley, W. 1963. Tractatus de obligationibus. In An introduction to the logical treatise 'De Obligationibus' with critical texts of William of Sherwood (?) and Walter Burley, Louvain, ed. R. Green.

Burley, W. 1988. Obligations (selections). In Cambridge translations of medieval philosophical texts, vol. 1: Logic and the philosophy of language, ed. N. Kretzmann, and E. Stump, 369-412. Cambridge: Cambridge University Press.

Cogan, E., S. Parsons, and P. McBurney. 2005. What kind of argument are we going to have today? In Proceedings of the Fourth International Joint Conference on Autonomous Agents and Multiagent Systems.

Cogan, E., S. Parsons, and P. McBurney. 2006. New types of inter-agent dialogues. In Argumentation in multi-agent systems, LNAI 4049, ed. S. Parsons, N. Maudet, P. Moraitis, and I. Rahwan, 154-168. Berlin: Springer.

de Rijk, L.M. 1974. Some thirteenth century tracts on the game of obligation. Vivarium 12(2): 94-123. de Rijk, L.M. 1975. Some thirteenth century tracts on the game of obligation II. Vivarium 13(1): 22-54. de Rijk, L.M. 1976. Some thirteenth century tracts on the game of obligation III. Vivarium 14(1): 26-49.

Dutilh Novaes, C. 2007. Formalizing medieval logical theories: Suppositio, consequentiae and obligationes. Logic, Epistemology, and the Unity of Science, vol. 7. Berlin: Springer.

Gabbay, D., and J. Woods. 2001. Non-cooperation in dialogue logic. Synthese 127(1-2): 161-186.

Girle, R.A. 1996. Commands in dialogue logic. In Practical reasoning: Proceedings of the First International Conference on Formal and Applied Practical Reasoning (FAPR 1996), LNAI 1085, ed. D.M. Gabbay, and H.J. Ohlbach, 246-260. Bonn, Germany: Springer

Hamblin, C.L. 1970. Fallacies. London: Methuen.

Hamblin, C. 1971. Mathematical models of dialogue. Theoria 37: 130-155.

Harel, D., D. Kozen, and J. Tiuryn. 2002. Dynamic logic. In Handbook of philosophical logic, 2nd ed., vol. 4, ed. D.M. Gabbay and F. Guenther. The Netherlands: Kluwer.

Karunatillake, N.C., N.R. Jennings, I. Rahwan, and P. McBurney. 2009. Dialogue games that agents play within a society. Artificial Intelligence 173: 935-981.

King, P. 1991. Medieval thought-experiments: The metamethodology of mediaeval science. In Thought experiments in science and philosophy, ed. T. Horowitz and G.J. Massey, 43-64. Lanham, MD: Rowman \& Littlefield.

King, P. 2004. Opposing and responding: Comments on Paul Spade. Preprint. http://individual.utoronto. ca/pking/presentations/Spade_Comments.pdf.

Lorenzen, P., and K. Lorenz. 1978. Dialogische Logik. Wissenschaftliche Buchgesellschaft. 
Matheson, M., and A. Ravenscroft. 2001. Evaluating and investigating learning through collaborative argumentation: An empirical study. Technical report DDRG-01-01. Milton Keynes: The Open University.

Maudet, N. 2003. Negotiating dialogue games. Autonomous Agents and Multi-Agent Systems 7: $229-233$.

McBurney, P., and S. Parsons. 2002. Games that agents play: A formal framework for dialogues between autonomous agents. Journal of Logic, Language and Information 11: 315-334.

McBurney, P., and S. Parsons. 2009. Dialogue games for agent argumentation. In Argumentation in artificial intelligence, ed. I. Rahwan, and G. Simari, 261-280. Berlin: Springer.

McBurney, P., S. Parsons, and M. Wooldridge. 2002. Desiderata for agent argumentation protocols. In Proceedings of AAMAS'02, July 15-19, 2002, Bologna, Italy, 402-409.

Ockham, W. 1974. Opera philosophica, vol I. St. Bonaventure: Franciscan Institute.

Parsons, S., M. Wooldridge, and L. Amgoud. 2002. An analysis of formal inter-agent dialogues. In Proceedings of AAMAS'02, July 15-19, 2002, Bologna, Italy, 394-401.

Parsons, S., M. Wooldridge, and L. Amgoud. 2003a. On the outcomes of formal inter-agent dialogues. In Proceedings of the 2nd International Conference on Autonomous Agents and Multi-Agent Systems, Melbourne.

Parsons, S., M. Wooldridge, and L. Amgoud. 2003b. Properties and complexity of some formal interagent dialogues. Journal of Logic and Computation 13(3): 347-376.

Prakken, H. 2005. Coherence and flexibility in dialogue games for argumentation. Journal of Logic and Computation 15(6): 1009-1040.

Prakken, H. 2006. Formal systems for persuasion dialogue. Knowledge Engineering Review 21(2): 163-188.

Ravenscroft, A., and M.P. Matheson. 2002. Developing and evaluating dialogue games for collaborative e-learning. Journal of Computer Assisted Learning 18(1): 93-101.

Sklar, E., and S. Parsons. 2004. Toward the application of argumentation-based dialogues for education. In Proceedings of the 3rd International Conference on Autonomous Agents and Multi-Agent Systems, ed. C. Sierra and E. Sonenberg.

Spade, P.V. 2000. Why don't medieval logicians ever tell us what they're doing? Or, what is this, a conspiracy?, preprint, http://pvspade.com/Logic/docs/Conspiracy.pdf.

Spade, P.V. 2008. Medieval theories of obligationes. In Stanford Encyclopedia of Philosophy, fall 2008 $e d n$, ed. E.N. Zalta http://plato.stanford.edu/archives/fall2008/entries/obligationes/.

Spade, P.V. 1993. Unpublished talk on obligationes presented at the Midwestern Division of the American Philosophical Association.

Uckelman, S.L. 2011a. Deceit and indefeasible knowledge: The case of dubitatio. Journal of Applied Non-Classical Logics 21(3/4): 503-519.

Uckelman, S.L. 2011b. A dynamic epistemic logic approach to modeling obligationes. In LIRa Yearbook, Institute for Logic, Language \& Computation, ed. D. Grossi, S. Minica, B. Rodenhäuser, and S. Smets, 147-172.

Uckelman, S.L. 2011c. Obligationes as formal dialogue systems. In STAIRS 2010: Proceedings of the Fifth Starting AI Researcher's Symposium, ed. T. Ågotnes, 341-354. IOS Press.

Uckelman, S.L. 2012. Interactive logic in the Middle Ages. Logic and Logical Philosophy (Forthcoming).

van Ditmarsch, H., W. van der Hoek, and B. Kooi. 2007. Dynamic epistemic logic. Synthese Library Series, vol. 337. Berlin: Springer.

Walton, D. 2000. The place of dialogue theory in logic, computer science, and communication studies. Synthese 123(3): 327-346.

Walton, D.N., and E.C.W. Krabbe. 1995. Commitment in dialogue: Basic concepts of interpersonal reasoning. Albany: State University of New York Press.

Yrjönsuuri, M. 1993. The role of casus in some fourteenth century treatises on sophismata and obligations. In Argumentationstheorie. Scholastische Forschungen zu den logischen und semantischen Regeln korrekten Folgerns, Brill, ed. K. Jacobi, 301-321.

Yrjönsuuri, M. 1994. Obligationes: 14th century logic of disputational duties, Acta Philosophica Fennica, vol. 55. Societatis Philosophia Fennica.

Yrjönsuuri, M. (ed.) 2001. Medieval formal logic. The Netherlands: Kluwer. 